\title{
ALINHAMENTO ESTRATÉGICO DA RESPONSABILIDADE SOCIAL CORPORATIVA: UM ESTUDO DE CASO NO SETOR BANCÁRIO BRASILEIRO
}

\author{
Simone Ruchdi Barakat* \\ simonebarakat@gmail.com \\ João Maurício Gama Boaventura* \\ jboaventura@usp.br \\ Edilson Fernandes Polo* \\ polo@usp.br \\ *Universidade de São Paulo - RS / Brasil
}

http://dx.doi.org/10.1590/1413-2311.076.55866

Recebido em 24/05/2015

Aprovado em 16/02/2017

Disponibilizado em 31/05/2017

Avaliado pelo sistema "double blind review"

Revista Eletrônica de Administração

Editora-chefe: Andrea Oltramari

ISSN 1413-2311 (versão "on line")

Editada pela Escola de Asministração da Universidade Federal do Rio Grande do Sul

Periodicidade: Quadrimestral

Sistema Requerido: Adobe Acrobat Reader

\section{RESUMO}

A maioria dos estudos que buscou testar a relação entre desempenho social corporativo (CSP) e desempenho financeiro corporativo (CFP) indica que há uma relação positiva entre os dois desempenhos, porém não existe ainda consenso entre os pesquisadores. Uma forma de elucidar essa questão é analisar com maior profundidade as variáveis que podem intervir nessa relação. Uma das variáveis é o alinhamento estratégico da Responsabilidade Social Corporativa (RSC), uma vez que diversos autores têm defendido que o simples engajamento em atividades consideradas socialmente responsáveis não leva a geração de benefícios à empresa, mas sim quando elas estão alinhadas à estratégia organizacional. Diante desse contexto, o presente estudo buscou descrever os processos e práticas empresariais que favorecem o alinhamento estratégico da RSC. Para isso, foi proposto um framework, a partir da literatura, com as condições para o alinhamento e, em seguida, foi feito um estudo de caso de uma instituição financeira, escolhida pelos critérios de alto desempenho social e financeiro. 
Os resultados mostraram que os processos e práticas que favorecem o alinhamento são: melhoria do contexto competitivo, escolha de questões ligadas à atividade principal, diálogo com stakeholders, envolvimento da alta gestão, envolvimento das áreas funcionais e monitoramento dos resultados. O estudo contribui para o aprofundamento da discussão sobre a relação entre CSP e CFP com a criação de um modelo para análise do alinhamento estratégico da RSC.

Palavras-chave: Responsabilidade Social Corporativa; Alinhamento Estratégico; Setor Financeiro.

\title{
STRATEGIC ALIGHMENT OF CORPORATE SOCIAL RESPONSIBILITY: A CASE STUDY IN THE BRAZILIAN BANKING SECTOR
}

\begin{abstract}
Most studies that sought to test the relationship between corporate social performance (CSP) and corporate financial performance (CFP) indicates that there is a positive relationship between the two performances, but there is still no consensus among researchers. One way to elucidate this question is to analyze in greater depth the variables that can intervene in this relationship. One of these variables is the strategic alignment of Corporate Social Responsibility (CSR), since several authors have argued that the simple engagement in activities considered socially responsible does not lead to benefits to the company, but when they are aligned with the organizational strategy. In this context, the study aims to describe organizational processes and practices that favor the strategic alignment of CSR. For this, a framework was proposed, based on the literature, with the conditions for the alignment and then a case study of a financial institution selected by the high social and financial performance criteria was made. The results showed that the processes and practices that favor alignment are: improving the competitive environment, choice of issues related to the main activity, dialogue with stakeholders, involvement of top management, involvement of functional areas and monitoring the results. The study contributes to the further discussion on the relationship between CSP and CFP by the creation of a framework to analyze the strategic alignment of CSR.
\end{abstract}

Keywords: Corporate Social Responsibility; Strategic Alignment; Financial sector. 


\section{ALINEACIÓN ESTRATÉGICA DE RESPONSABILIDAD SOCIAL CORPORATIVA: UM ESTUDIO DE CASO EM EL SECTOR BANCARIO BRASILEÑO}

\section{RESUMEN}

La mayoría de los estudios que trataron de probar la relación entre el desempeño social de las empresas (CSP) y su desempeño financiero (CFP) indica que hay una relación positiva entre los dos desempeños, pero todavía no hay consenso sobre el tema entre los investigadores. Una manera de dilucidar esta cuestión es analizar con mayor profundidad las variables que pueden intervenir de esta relación. Una de las variables es la alineación estratégica de la Responsabilidad Social Empresarial (RSE), ya que muchos autores han argumentado que simplemente participar en actividades consideradas socialmente responsables no conduce a la generación de rentas para la empresa, pero solamente cuando están alineadas con la estrategia de la organización. En este contexto, este estudio tiene como objetivo describir los procesos y las prácticas organizacionales que favorecen la alineación estratégica de la RSE. Para esto, se ha propuesto modelo, enbasado en la literatura, relacionando las condiciones para el alineamiento y luego se hizo un estudio de caso de una institución financiera seleccionada por los criterios de alto desempeño social y financeiro. Los resultados indicaran que los procesos y prácticas que favorecen la alineación son: la mejora del entorno competitivo, la selección de temas relacionados con la actividad principal, el diálogo con las partes interesadas, la participación de la alta dirección y de las áreas funcionales y monitorización de los resultados. El estudio contribuye a la discusión sobre la relación entre CSP y CFP por la creación de un modelo para analizar la alineación estratégica de la RSE.

Palabras Clave: Responsabilidad Social Empresarial; Alineación estratégica; Sector financiero.

\section{INTRODUÇÃO}

Nos últimos trinta anos, estudos realizados com o objetivo de testar a relação entre desempenho social corporativo (CSP) e desempenho financeiro corporativo (CFP) têm dominado a pesquisa em Responsabilidade Social Corporativa (RSC) (CRANE et al., 2008). Por um lado, alguns autores defendem que a adoção de práticas socialmente responsáveis 
melhora o desempenho financeiro da empresa (CRANE et al., 2008; McWILLIAMS; SIEGEL, 2011), pois trazem benefícios como redução riscos e passivos socioambientais (MARCONDES; BACARJI, 2010), licença social para operar (KURUCZ, 2008) e bom clima organizacional (FOMBRUN; GARDBERG; BARNETT, 2000; BRANCO; RODRIGUES, 2006). Por outro lado, há a visão de que essas práticas geram custos que as empresas nunca vão recuperar (FRIEDMAN, 1970; RUSSO; FOUTS, 1997; KARNANI, 2011).

Autores que realizaram uma meta análise desses estudos encontraram que há uma relação positiva entre CSP e CFP (ORLITZKY; SCHMIDT; RYNE, 2003; MARGOLIS; WALSH, 2003), porém não se pode afirmar que haja uma relação direta entre eles. Mesmo com o cuidado de controlar as variáveis que podem intervir nessa relação, é muito difícil provar estatisticamente uma relação causal entre os esses dois desempenhos. Muitos fatores endógenos podem levar à adoção de práticas de RSC, por exemplo, relacionados à inovação, recursos humanos, reputação e cultura organizacional (SURROCA; TRIBÓ; WADDOCK, 2010). Esses fatores influenciam tanto a CSP quanto a CFP, o que poderia confundir os resultados. Além disso, a própria CFP pode influenciar a adoção de práticas de RSC, invertendo-se a direção da relação causal.

Uma forma de elucidar essa discussão é analisar com maior profundidade as variáveis que podem intervir na relação entre CSP e CFP. Uma dessas variáveis é o alinhamento estratégico da RSC, uma vez que diversos autores (BURKE; LOGSDON, 1996; PORTER; KRAMER, 2002, 2006; McWILLIAMS; SIEGEL, 2001, 2011; McWILLIAMS; SIEGEL; WRIGHT 2006; HUSTED; SALAZAR, 2006; MOLTENI, 2006; NASCIMENTO; LEMOS; MELLO, 2008) têm defendido que o simples engajamento em atividades consideradas socialmente responsáveis não leva à geração de benefícios à empresa, mas sim quando estão alinhadas à estratégia organizacional. Em outras palavras, a RSC levaria à criação de valor para empresa quando alinhada à estratégia organizacional.

Diante dessa discussão, o presente estudo buscou verificar quais processos e práticas empresarias favorecem o alinhamento estratégico da RSC. Para atingir esse objetivo, foi proposto um modelo de alinhamento estratégico baseado na literatura acadêmica sobre o tema e, em seguida, o modelo foi aplicado empiricamente em uma empresa do Setor Bancário Brasileiro que apresenta alta CSP e alta CFP. 


\section{FUNDAMENTAÇÃO TEÓRICA}

\subsection{Responsabilidade Social Corporativa (RSC)}

A RSC é ainda considerada um campo de pesquisa em desenvolvimento. DeBakker, Groenwegen e Den Hond (2005) realizaram uma análise bibliométrica de mais de 500 artigos sobre RSC publicados nos últimos 35 anos e concluíram que não é possível evidenciar uma operacionalização dos conceitos gerais centrais. O desenvolvimento do construto de RSC é caracterizado por três aspectos distintos: progressão, variedade e normativismo. A progressão significa que o construto foi desenvolvido a partir da teoria empiricamente testada. A variedade explica que o conceito alterna seu significado de acordo com o contexto ou com a relação que estabelece com temas complementares. E, finalmente, o normativismo refere-se ao caráter prescritivo encontrado nos trabalhos nesse campo (DeBAKKER; GROENWEGEN; DEN HOND, 2005).

Da mesma forma, Lockett, Moon e Visser (2006, p. 113) concluíram, a partir de um estudo sobre a literatura de RSC, em um período de dez anos, que o conhecimento sobre o tema pode melhor ser descrito como "em estado contínuo de emergência". Segundo esses autores, o campo não é caracterizado pelo domínio de uma abordagem teórica, método e pressupostos particulares, é um campo sem "paradigma".

Portanto, é possível identificar na literatura uma grande variedade de conceitos e premissas sobre o que deve abranger a responsabilidade social da empresa (CARROLL, 2008). Uma das primeiras tentativas de formalizar o significado de RSC foi feita por Keith Davis, que argumentou que RSC refere-se a "decisões e ações dos homens de negócios baseadas, pelo menos em parte, além dos interesses diretamente econômicos e técnicos da empresa" (DAVIS, 1960, p.70). Além disso, afirmou que algumas decisões socialmente responsáveis podem, no longo prazo e mediante um processo complexo, trazer ganhos econômicos para a empresa. Na mesma época, Frederick (1960) afirmou que a responsabilidade social implica em uma postura pública com relação aos recursos econômicos e humanos da sociedade, e uma disposição em ver esses recursos utilizados para fins sociais mais amplos e não somente para interesses de poucos. Nas décadas seguintes, diversos outros autores elaboraram definições 
para o conceito de RSC (CARROLL, 1979, 1991; JONES, 1980; MICHALOS, 1997 entre outros).

No presente estudo, adotou-se a definição de Jones (1980), na qual RSC é a responsabilidade da empresa com outros grupos da sociedade, além dos acionistas, e que vão além daquelas prescritas pela lei e por contratos. Nessa definição, a RSC só existe se for adotada voluntariamente pela empresa e se estende a grupos como consumidores, funcionários, fornecedores, governo e comunidades locais.

Com o objetivo de medir a RSC da empresa, trabalhos teórico-empíricos utilizam a CSP como construto. A mensuração da CSP considera o nível em que os diversos stakeholders da organização são atendidos em suas demandas (CLARKSON, 1995). A CSP reflete a ideia de que as responsabilidades são parte integrante das ações, decisões, comportamentos e impactos corporativos (SURROCA; TRIBÓ; WADDOCK, 2010). Em outras palavras, o conceito de CSP refere-se à resposta da organização frente às demandas dos diversos grupos com os quais tem responsabilidades (ULLMANN, 1985). Nesse sentido, melhorar CSP significa alterar o comportamento organizacional para produzir menos danos e resultados mais benéficos para a sociedade (WOOD, 1991).

Para o presente estudo, considera-se CSP como o amplo conjunto de processos e práticas que a empresa desenvolve para lidar e criar relacionamentos com os seus inúmeros stakeholders e com o meio ambiente (WADDOCK, 2004).

\subsection{Alinhamento Estratégico da RSC}

O conceito de alinhamento estratégico tem origem na obra clássica de Chandler, "Strategy and Structure", de 1962. Na ocasião, Chandler constatou a existência de uma forte correlação entre estrutura da empresa e sua estratégia. O autor observou que a estrutura da empresa, com o passar do tempo, se ajustava à sua respectiva estratégia.

Atualmente, sabe-se que diversas outras dimensões das organizações, além da estrutura, influenciam o alinhamento estratégico, a exemplo do capital humano, capital da informação e cultura organizacional (KAPLAN; NORTON, 2004). O conceito de alinhamento estratégico é 
de adequação das diversas dimensões da organização com a sua estratégia e uma forma apropriada para analisá-lo é identificar as diversas dimensões organizacionais que possam ou precisam ser modificadas para terem conformidade com a estratégia.

No presente estudo, a dimensão organizacional analisada é a RSC. Seguindo a mesma lógica do alinhamento estratégico, Nascimento, Lemos e Mello (2008, p. 18) afirmam que a responsabilidade social corporativa estratégica é a inserção da variável socioambiental ao longo de todo o processo gerencial de planejar, organizar, dirigir e controlar, utilizando-se das funções organizacionais como marketing, produção, finanças, recursos humanos e outras, bem como das interações que ocorrem no ecossistema do mercado, visando a atingir os objetivos e metas organizacionais.

É possível observar na literatura um conjunto de condições levantadas para o alinhamento estratégico da RSC. Mais especificamente, observou-se a menção das seguintes condições: 1) melhoria do contexto competitivo, 2) escolha de questões ligadas à atividade principal da empresa, 3) diálogo com stakeholders e 4) monitoramento e gerenciamento dos resultados. Essas condições serão, a seguir, explicadas.

A primeira delas é a utilização das práticas sociais e ambientais para melhorar o contexto competitivo. O contexto competitivo é a qualidade do ambiente de negócios em que a empresa opera e sempre foi importante para a estratégia. Variáveis contextuais, como: disponibilidade de funcionários capacitados e motivados; eficiência da infraestrutura local; tamanho e sofisticação do mercado local; escopo da regulamentação governamental, afetam a capacidade competitiva. Portanto, a empresa pode atingir o benefício social combinado com o benefício econômico ao investir na melhoria do contexto competitivo. Para isso, é preciso identificar áreas em que o valor social coincide com o econômico e que poderão beneficiar a competitividade da empresa e de seu polo (PORTER; KRAMER, 2002).

A segunda condição para o alinhamento é concentrar as iniciativas de RSC em questões que tenham alguma interseção com a área de atuação da empresa. Quanto maior o vínculo da questão com a atividade principal da empresa, maior será a oportunidade de alavancar recursos, tanto em benefício da sociedade quanto em benefício da empresa (PORTER; KRAMER, 2006). Em outras palavras, atividades com alto grau de centralidade e 
especificidade possuem maior probabilidade de gerar valor para tanto para empresa quanto para sociedade (BURKE; LOGSDON, 1996).

Outra condição trata-se do estabelecimento do diálogo com os stakeholders. Uma dificuldade que pode ser encontrada pela empresa é a identificação das expectativas dos seus stakeholders, uma vez que as repercussões de uma nova estratégia ainda não foram testadas. Para resolver essa dificuldade, a organização pode criar um diálogo preliminar com seus stakeholders, reforçando sua capacidade de prever os problemas sociais e ambientais (MOLTENI, 2006). Após a identificação das expectativas, é possível buscar soluções inovadoras para atendê-las, e a RSC pode se tornar um fator criativo para o desenvolvimento da competitividade da empresa.

Finalmente, uma condição importante trata-se da utilização de um modelo ou sistema de indicadores para avaliação dos resultados. A empresa passa a monitorar e gerenciar os dados na medida em que atribui importância e relevância ao tema que justifique o acompanhamento. Além disso, quanto mais alto o nível de gestão ao qual os dados são reportados, maior o indício da importância atribuída ao tema (CLARKSON, 1995). Portanto, para a RSC estar alinhada com a estratégia, espera-se que a empresa monitore e gerencie os dados relativos às questões de RSC, além de reportar os resultados a níveis gerenciais altos.

Além dessas condições levantadas na literatura de RSC, utilizou-se, no presente estudo, o modelo de alinhamento estratégico proposto por Henderson e Venkatraman (1989), em que o alinhamento é feito a partir da 1) integração estratégica, que refere-se à integração com a formulação e implementação da estratégia; e 2) integração funcional, que refere-se a integração com as outras áreas funcionais.

A partir desse levantamento, o presente estudo propõe um framework de análise do alinhamento estratégico da RSC, apresentado na Figura 1. De acordo com o modelo, a CSP leva à CFP caso a RSC esteja alinhada com a estratégia organizacional. As condições que favorecem o alinhamento foram buscadas na literatura de RSC e estão indicadas no modelo, as quais são: 1) melhora do contexto competitivo; 2) vínculo das práticas de RSC com a atividade principal da empresa; 3) diálogo com stakeholders e 4) monitoramento e gerenciamento de resultados. Além dessas, foram colocadas no modelo as seguintes 
condições: 5) envolvimento da alta gestão e 6) envolvimento das áreas funcionais, baseadas no modelo de alinhamento estratégico de Henderson e Venkatraman (1989).

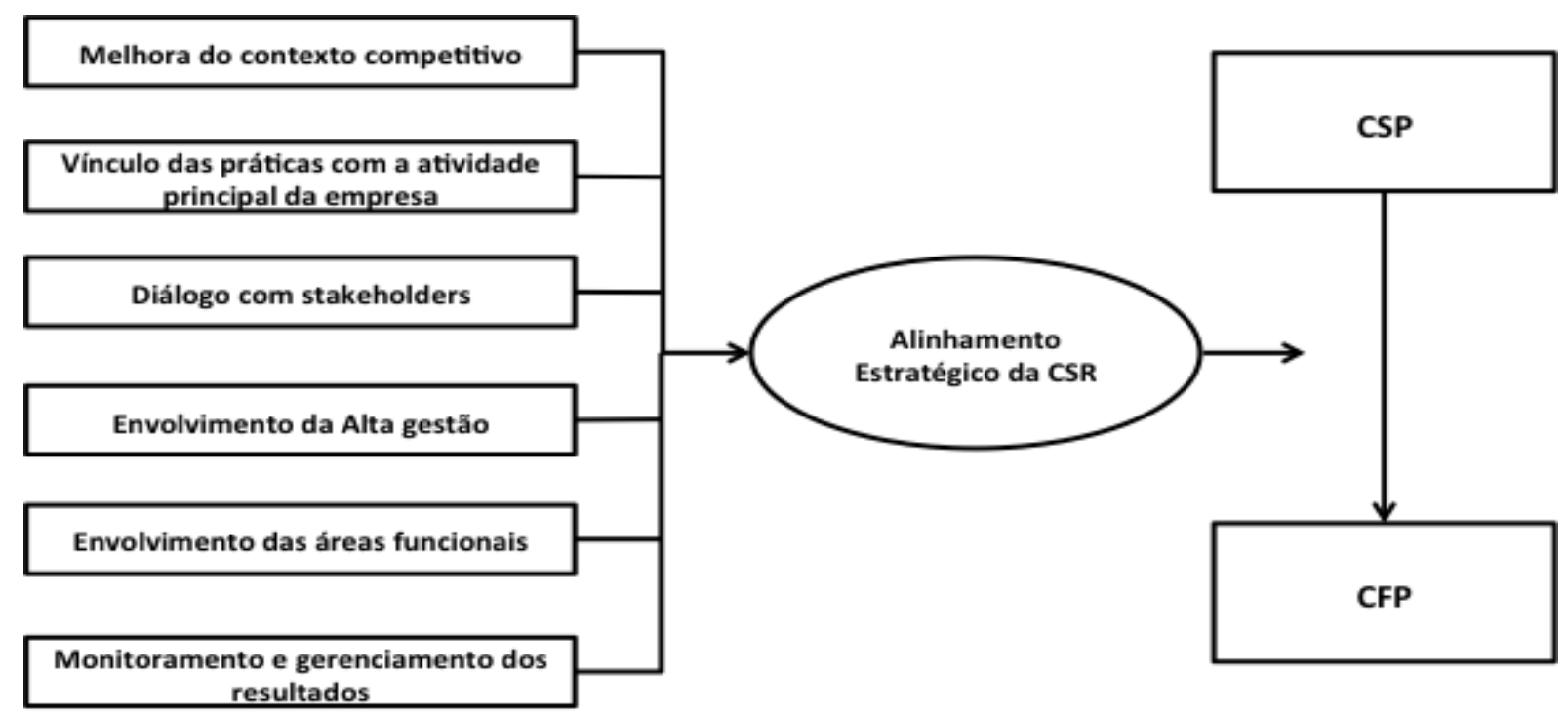

Figura 1: Framework para análise do alinhamento estratégico da RSC.

\section{PROCEDIMENTOS METODOLÓGICOS}

Para atingir o objetivo de descrever os processos e práticas empresariais que favorecem o alinhamento estratégico da RSC, optou-se pela utilização de uma abordagem qualitativa por meio do Estudo de Caso, pois, conforme identificado na literatura, o campo da RSC é pouco consolidado e abrange uma série de elementos e relações complexas, as quais poderão ser mais bem analisadas com um estudo em maior profundidade e que considere o fenômeno na sua totalidade.

A abordagem qualitativa envolve entrevistas e observações que não resultam em dados quantitativos, mas em descrições dos resultados e das impressões dos eventos. Com essa abordagem, é possível explorar relações que não poderiam ser feitas de outra forma. A maior limitação é a impossibilidade de generalizações dos resultados para populações maiores ou para situações mais gerais. Normalmente, não é possível incluir amostras representativas, devido problemas práticos de escala e à natureza das observações e das situações estudadas (BLACK, 1999). 
A partir da fundamentação teórica apresentada no capítulo anterior, elaborou-se o Quadro 1, que traz as dimensões analisadas no presente estudo, os elementos que nortearam o estudo e os respectivos autores que os fundamentam.

Quadro1 - Diretrizes teóricas da pesquisa.

\begin{tabular}{|c|c|c|}
\hline Dimensão & Elementos norteadores & $\begin{array}{c}\text { Fundamentação Teórica } \\
\text { (Autores) }\end{array}$ \\
\hline $\begin{array}{c}\text { Responsabilidade } \\
\text { Social Corporativa } \\
\text { (RSC) }\end{array}$ & $\begin{array}{l}\quad \text { Definição de RSC; } \\
\text { - } \quad \text { Questões que envolvem RSC; } \\
\text { - } \quad \text { Públicos aos quais a RSC se dirige. }\end{array}$ & $\begin{array}{c}\text { Carroll (1979, 1991, 1999, 2008); } \\
\text { DeBakker, Groenwegen e Den } \\
\text { Hond (2005); Lockett, Moon e } \\
\text { Visser (2006); Crane } \text { et al. }(2008)\end{array}$ \\
\hline $\begin{array}{l}\text { Desempenho Social } \\
\text { Corporativo (CSP) }\end{array}$ & $\begin{array}{l}\text { - Forma como a organização responde às } \\
\text { - } \text { questões de RSC; } \\
\text { - } \quad \text { Infividades e processos realizados; } \\
\text { - atividades utilizadas para a escolha das } \\
\text { - Envolvimento das áreas funcionais. }\end{array}$ & $\begin{array}{c}\text { Wood (1991); Clarkson (1995); } \\
\text { Nascimento, Lemos e Mello } \\
\text { (2008); Surroca, Tribó e Waddock } \\
\text { (2010) }\end{array}$ \\
\hline $\begin{array}{l}\text { Alinhamento entre } \\
\text { RSC e Estratégia } \\
\text { Organizacional }\end{array}$ & $\begin{array}{l}\text { - Inserção da variável sociambiental no } \\
\text { processo formal de formulação } \\
\text { estratégica; } \\
\text { - } \quad \text { Vínculo das práticas socioambientais } \\
\text { com a atividade principal da empresa; } \\
\text { - } \quad \text { Disseminação da RSC na organização; } \\
\text { - } \quad \text { Forma de avaliação dos resultados - } \\
\text { utilização de modelo ou sistema de } \\
\text { indicadores para avaliaçãao do } \\
\text { desempenho social; } \\
\text { - Nível organizacional ao qual os } \\
\text { resultados são reportados. }\end{array}$ & $\begin{array}{l}\text { Hart (1995, 1997); Burke e } \\
\text { Logsdon (1996); Fombrun, } \\
\text { Gardberg e Barnett (2000); Husted } \\
\text { (2000); McWilliams e Siegel } \\
\text { (2000); Husted e Allen (2001); } \\
\text { Porter e Kramer (2002, 2006); } \\
\text { Husted e Salazar, (2006); Molteni } \\
\text { (2006); McWilliams, Siegel e } \\
\text { Wright (2006, 2011); Nascimento, } \\
\text { Lemos e Mello (2008) }\end{array}$ \\
\hline
\end{tabular}

\subsection{Seleção do Caso}

Em Estudo de Caso único, é razoável escolher uma empresa em que o processo de interesse seja facilmente observável e que seja apropriado para iluminar e ampliar relações e lógicas entre construtos (EISENHARDT; GRAEBNER, 2007). No caso do presente estudo, o processo de interesse são as práticas e processos empresariais realizados para o alinhamento estratégico da RSC. Por isso, foi escolhida uma empresa com alta CSP, pois a alta CSP implica em presença de práticas e processos de RSC, sem os quais não seria possível estudar o alinhamento. Ao mesmo tempo, a empresa deveria ter alta CFP, uma vez que, de acordo com a literatura, a falta de alinhamento estratégico da RSC poderia comprometer os resultados financeiros. Portanto, conforme mostrado no modelo proposto pelo presente estudo (Figura 1), espera-se que empresas que possuam alta CSP e alta CFP tenham processos e práticas que favoreçam o alinhamento estratégico da RSC. 
Como critério de alta CSP, foram utilizadas as seguintes informações:

a) ter participado das treze edições da carteira do Dow Jones Sustainability Index (DJSI), desde quando o índice foi criado, em 1999. O DJSI é formado por um conjunto de índices que avaliam o desempenho socioambiental das 2500 maiores empresas listadas no Dow Jones Global Total Stock Market;

b) ter participado das oito edições da carteira do Índice de Sustentabilidade Empresarial (ISE), da BM\&FBOVESPA, desde a criação do índice em 2005. O ISE é um índice de sustentabilidade criado pela Bolsa de Valores de São Paulo e o Centro de Estudos em Sustentabilidade da Fundação Getúlio Vargas (GVces), com financiamento do International Finance Corporation (IFC), órgão de financiamento privado do Banco Mundial.

Como critério de alta CFP, foram consideradas informações financeiras da empresa. A empresa escolhida foi classificada como o segundo banco privado mais rentável, em 2013, entre instituições da América Latina e dos Estados Unidos, de capital aberto com ativos superiores a US\$ 100 bilhões. Essa classificação foi realizada a partir da rentabilidade sobre o patrimônio (ROE) nos últimos 12 meses dos bancos que, no final de junho de 2013, tinham ativos superiores a US\$ 100 bilhões (ECONOMÁTICA, 2013).

De acordo com esse estudo, o banco escolhido para o presente estudo apresentou ROE de 16,83\% e é o banco privado com mais ativos da América Latina (US\$ 437,384 no final de julho de 2013), valor acima da rentabilidade média dos quatro maiores bancos brasileiros, que foi de 16,4\% no mesmo período (ECONOMÁTICA, 2013).

\subsection{Coleta e Análise de Dados}

Para atingir o objetivo do estudo, foram coletados dados secundários extraídos dos relatórios divulgados pela empresa: Relatórios Anuais de Sustentabilidade de 2008 a 2012. Foram coletados também dados secundários de documentos e relatórios elaborados por outras instituições ligadas ao setor financeiro: Instituto Ethos, Banco Central do Brasil (BCB), Federação Brasileira de Desenvolvimento Sustentável (FBDS), IFC, BankTrack e Amigos da 
Terra, para identificar práticas de RSC do setor, decorrentes de acordos voluntários e regulamentações.

Foram coletados, também, dados primários por meio de entrevistas semiestruturadas individuais. Foram entrevistadas a superintendente de sustentabilidade, uma gerente de sustentabilidade e uma gerente de cultura e clima organizacional. As gestoras de Sustentabilidade foram selecionadas pois possuem alto nível gerencial, participam dos processos de decisões e têm acesso a todas as informações relativas ao fenômeno estudado. A gerente de cultura e clima organizacional foi escolhida por entender-se que seria necessário um entrevistado fora da área de sustentabilidade, que pudesse ter informações sobre a disseminação do tema pelas outras áreas da organização. As entrevistas foram realizadas no período de 01/04/2013 a 03/06/2013. As entrevistadas foram denominadas E1, E2 e E3.

Uma vez que nem todos os aspectos de um caso podem ser compreendidos, a decisão de até onde investigar é uma escolha do pesquisador (STAKE, 2005). Dessa forma, o número de entrevistas foi escolhido devido ao entendimento de que mais entrevistados não trariam informações novas relevantes sobre o fenômeno de interesse do estudo.

Os processos e práticas analisados no estudo, as respectivas questões investigativas e as fontes de dados utilizadas para análise são mostradas, a seguir, no Quadro 2.

Quadro 2 - Processos e práticas analisados no estudo.

\begin{tabular}{|c|c|c|}
\hline $\begin{array}{l}\text { Processos e } \\
\text { Práticas }\end{array}$ & Questões investigativas & Fontes de Dados \\
\hline $\begin{array}{l}\text { Melhoria do } \\
\text { contexto competitivo }\end{array}$ & $\begin{array}{l}\text { As práticas de RSC estão relacionadas com melhoria do } \\
\text { contexto competitivo: disponibilidade de funcionários } \\
\text { capacitados e motivados; eficiência da infraestrutura local; } \\
\text { tamanho e sofisticação do mercado local; escopo da } \\
\text { regulamentação governamental? }\end{array}$ & $\begin{array}{l}\text { Relatórios de } \\
\text { Sustentabilidade, } \\
\text { Relatórios de } \\
\text { outras instituições } \\
\text { e entrevistas. }\end{array}$ \\
\hline $\begin{array}{l}\text { Vínculo da RSC com } \\
\text { a atividade principal } \\
\text { da empresa }\end{array}$ & $\begin{array}{l}\text { Existe vínculo entre as práticas e a atividade principal da } \\
\text { empresa? } \\
\text { Quais critérios são utilizados para escolha das práticas? }\end{array}$ & $\begin{array}{c}\text { Relatórios de } \\
\text { Sustentabilidade e } \\
\text { Entrevistas }\end{array}$ \\
\hline $\begin{array}{l}\text { Diálogo com } \\
\text { stakeholders }\end{array}$ & $\begin{array}{l}\text { A quais públicos a empresa considera que a RSC se dirige } \\
\quad \text { (interno, externo, ambos)? } \\
\text { Como as expectativas dos stakeholders são identificadas? }\end{array}$ & $\begin{array}{c}\text { Relatórios de } \\
\text { Sustentabilidade e } \\
\text { Entrevistas }\end{array}$ \\
\hline $\begin{array}{l}\text { Envolvimento das } \\
\text { áreas funcionais }\end{array}$ & $\begin{array}{c}\text { Há uma definição formalizada sobre RSC? } \\
\text { O entendimento sobre RSC é difundido por toda } \\
\text { organização? } \\
\text { Como as áreas funcionais da empresa são envolvidas? }\end{array}$ & Entrevistas \\
\hline
\end{tabular}




\begin{tabular}{|c|c|c|}
\hline $\begin{array}{c}\text { Envolvimento da } \\
\text { Alta gestão }\end{array}$ & $\begin{array}{c}\text { Como os líderes da organização lidam com o tema? } \\
\text { Quais níveis organizacionais estão envolvidos com o tema? } \\
\text { A qual nível organizacional os resultados são reportados? } \\
\text { Qual a frequência? }\end{array}$ & Entrevistas \\
\hline $\begin{array}{c}\text { Monitoramento e } \\
\text { gerenciamento dos } \\
\text { resultados }\end{array}$ & $\begin{array}{c}\text { É utilizado algum modelo ou sistema para elaboração de } \\
\text { relatórios de RSC? Caso sim, quais? } \\
\text { Como é feita a avaliação dos resultados? }\end{array}$ & Entrevistas \\
\hline
\end{tabular}

\section{RESULTADOS}

Nesta sessão, os resultados do estudo de caso são apresentados e discutidos. Primeiramente, foi feito um levantamento sobre a RSC no Setor Bancário e a RSC nos Bancos Brasileiros. Esse levantamento foi feito para que o caso pudesse ser devidamente analisado considerandose os movimentos de práticas, regulações e acordos que vêm ocorrendo no setor bancário. Em seguida, os processos e práticas da empresa estudada foram analisados a luz do modelo de alinhamento estratégico da RSC proposto por esse estudo.

\subsection{RSC no Setor Bancário}

Tradicionalmente, os impactos sociais e ambientais das empresas de serviços não são considerados tão relevantes quanto os impactos dos setores produtivos. Porém, por sua importância na economia, o setor financeiro, que tem contato com todos os outros setores produtivos, tem um enorme potencial para influenciar mudanças na sociedade. Além disso, os bancos estão expostos a um conjunto de questões sociais e ambientais mais diverso do que a maioria dos negócios, por meio das carteiras dos seus clientes.

As práticas de responsabilidade socioambiental também oferecem oportunidades para as instituições financeiras melhorarem os seus próprios produtos e serviços. Os bancos e outras instituições financeiras estão, cada vez mais, se deslocando da postura de evitar riscos para a postura de criar oportunidades, ou seja, saindo da postura defensiva- em que a questão social e ambiental é vista como um custo adicional - para a postura sustentável - em que a responsabilidade social é vista como uma vantagem e uma oportunidade de crescimento (IFC, 2007).

Portanto, para as instituições financeiras, a responsabilidade socioambiental tem dois componentes: 1) gestão dos riscos sociais e ambientais na tomada de decisões estratégicas e 
na concessão de empréstimos; e 2) identificação de oportunidades para o desenvolvimento de novos produtos e serviços em áreas relacionadas à responsabilidade socioambiental, por exemplo, eficiência energética, tecnologias de produção mais limpas, microfinanças e serviços financeiros voltados para habitação de baixa renda.

\subsection{RSC nos Bancos Brasileiros}

As primeiras ações dos bancos brasileiros voltadas para a RSC podem ser identificadas, no início de 1990, quando o Banespa publicou o primeiro relatório social do Sistema Financeiro Nacional (SFN). Em 1993, a Federação Brasileira de Bancos (FEBRABAN) emitiu o primeiro relatório social para a indústria financeira. E, desde 2004, cinco dos maiores bancos no Brasil têm disponibilizado informações não financeiras para o público, seja por meio de relatório social ou em seus relatórios anuais.

Em 2001, o então banco líder de renome mundial em sustentabilidade, Banco Real, começou a incorporar formalmente avaliações de aspectos sociais e ambientais das operações em seu processo de aprovação de financiamento. Em 2000, o Banco BBA Creditanstalt (hoje Banco Itaú-BBA) tornou-se o primeiro banco a estabelecer um conjunto de procedimentos formais para gerenciar os riscos sociais e ambientais, e exigir a consideração explícita dessas questões em financiamentos de projetos. Em 2002, o Unibanco lançou o seu próprio sistema de gerenciamento de risco social e ambiental.

Com relação a acordos voluntários, o Brasil foi um dos primeiros países a desenvolver um conjunto de princípios para as instituições que operam no país, o Protocolo Verde, e é um dos países com maior número de instituições signatárias de padrões internacionais. Grandes bancos brasileiros são referências reconhecidas internacionalmente. Por exemplo, o Itaú Unibanco faz parte do DJSI desde 1999, e o Bradesco desde 2006.

Alguns bancos brasileiros também foram reconhecidos internacionalmente em premiações e relatórios importantes relativos ao tema. O Banco $A B N$ AMRO Real ganhou o prêmio Sustainable Finance Awards, concedido pelo jornal britânico Financial Times e pelo IFC, de banco mais sustentável de países emergentes, em 2006. Além disso, o ABN AMRO Real foi um estudo de caso da Harvard Business School pela aplicação de critérios de nível 
internacional na integração de aspectos sociais e ambientais por toda a instituição. O Banco do Brasil ganhou o prêmio em 2007, na categoria de países latino-americanos. Além disso, os bancos Itaú BBA e Unibanco já foram citados como exemplos de atuação responsável em relatórios do IFC sobre sustentabilidade bancária em países emergentes. Em 2011, o Itaú Unibanco foi eleito o banco mais sustentável do mundo pelo mesmo prêmio.

Com relação à regulamentação das práticas de RSC, apenas mais recentemente, alguns órgãos como o BCB vêm criando mecanismos para incluir a responsabilidade socioambiental no SFN. Em 2008, o BCB determinou que os bancos públicos e privados que fornecem crédito rural devem exigir documentação comprobatória de regularidade ambiental para financiamentos agropecuários na região amazônica. Em meados de 2010, a Diretoria Colegiada do BCB criou uma área no Departamento de Análise de Atos Normativos (DENOR) para tratar dos assuntos relacionados à responsabilidade socioambiental do SFN e da inclusão financeira da população. Ainda em 2010, foi implementado o Projeto Responsabilidade Socioambiental do SFN, que estabelece um padrão de boas práticas para instituições financeiras reguladas pelo BCB (BANKTRACK; AMIGOS DA TERRA, 2012).

Contudo, somente em 2012, o BCB se posicionou como regulamentador, ao publicar o Edital de Audiência Pública 41/2012, para tornar obrigatória a implementação de uma política de responsabilidade socioambiental por todas as instituições financeiras e outros órgãos. A medida também prevê que as companhias abertas devem necessariamente publicar um relatório socioambiental.

É possível notar, portanto, que apesar escassa, a regulamentação com relação à responsabilidade socioambiental dos bancos vem aumentando nos últimos anos. As novas medidas têm buscado enfatizar o gerenciamento dos riscos de natureza socioambiental, obrigando os bancos a possuir ferramentas para a mensuração desses riscos em suas atividades e criando um sistema de avaliação com critérios consistentes.

\subsection{RSC no Caso Estudado}

A análise do caso estudado foi feita conforme os processos e práticas ilustrados no modelo de alinhamento estratégico da RSC proposto pelo estudo (Figura 1). Cada prática foi analisada 
com base nas questões investigativas e relacionada com a fundamentação teórica, conforme mostrado na Figura 3.

3.3.1 Práticas relacionadas à melhoria do contexto competitivo e à atividade principal da empresa.

Os temas escolhidos como prioritários, atualmente, para a empresa, são: educação financeira; riscos e oportunidades socioambientais; diálogo e transparência. Essa escolha foi realizada, em 2011, quando os processos e práticas de RSC foram redesenhados com o objetivo de integrar a RSC à agenda de negócios. A entrevistada E1 explica que essa escolha muda com o tempo, pois as variáveis que afetam o negócio são muito dinâmicas e precisam estar sendo sempre revistas. Os gestores estão, constantemente, questionando qual é o melhor jeito de utilizar os recursos disponíveis e de influenciar uma mudança. As respostas para esses questionamentos não duram por um prazo longo. Por isso, a questão principal é a atuação na gestão da empresa e não a busca de uma política definitiva do que deve ser feito. A entrevistada ainda afirma que, para se manter na liderança, é preciso estar preparado para ler as mudanças e interagir com elas e não reagir a elas.

A mudança na escolha dos temas prioritários mostra que a empresa passou a lidar com a RSC a partir de questões ligadas à sua atividade principal, conforme defendido por Porter e Kramer (2006). A Figura 2 mostra a mudança na escolha dos temas prioritários. Antes, em 2009, havia oito temas considerados prioritários, os quais eram bastante diversos e, muitas vezes, dissociados da atividade principal da empresa. Depois, a partir de 2011, a empresa escolheu apenas três temas, considerados estratégicos. 


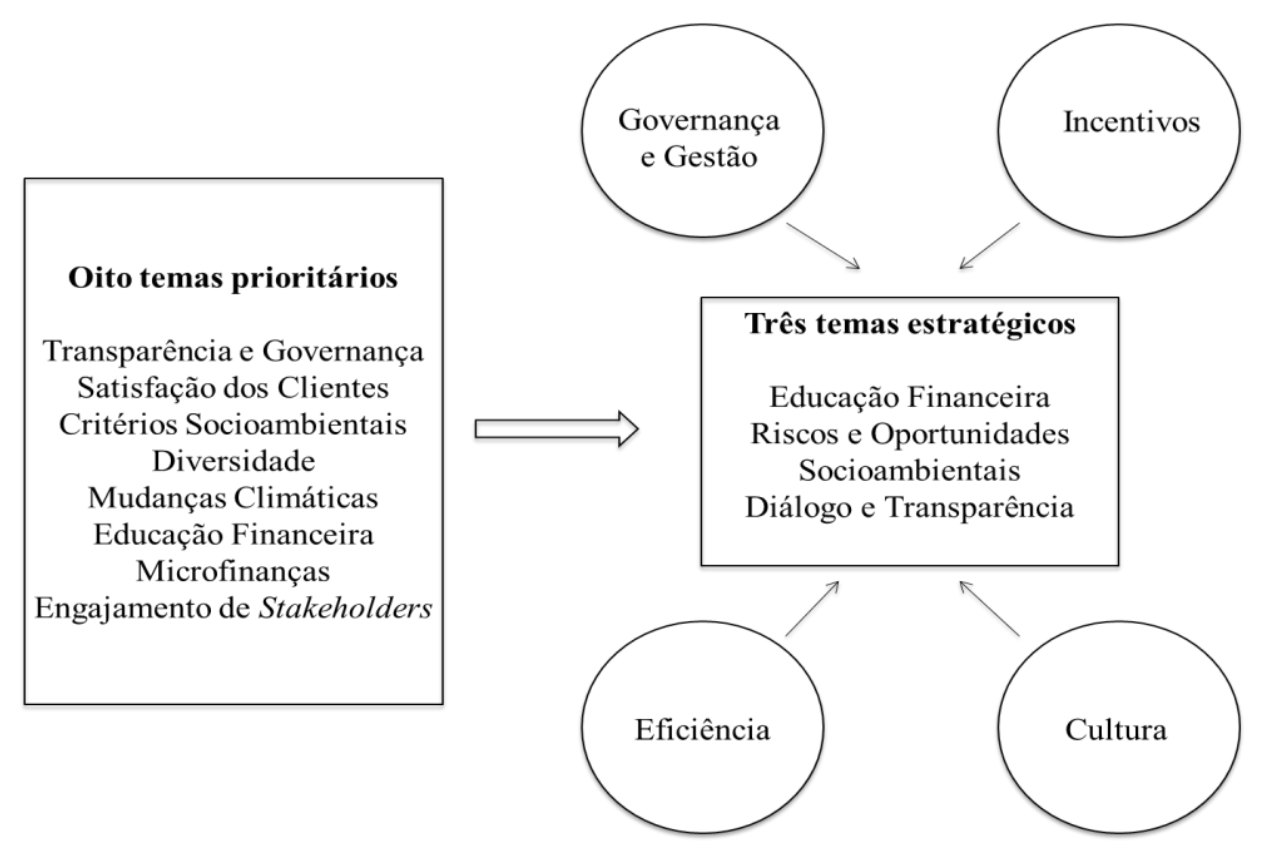

Figura 2 - Mudança dos temas prioritários de RSC.

FONTE: Baseado em Relatório de Sustentabilidade, 2012.

A entrevistada E1 destaca a importancia das escolhas e afirma que existe, por parte da empresa, um comprometimento e uma capacidade de avaliar se o que está sendo feito e proposto é adequado ou esperado.

Se, por exemplo, amanhã eu resolver que o melhor projeto é salvar as baleias do Ártico. Faz sentido? É ótimo para o planeta e até para as baleias, mas não tem nenhum sentido para mim. Então, ser coerente com aquilo que você faz no dia a dia é uma das variáveis importantes [...] A sabedoria está em escolher, se não você gasta um monte de recursos e não muda nada. Então estratégia é fundamental. É por isso que a gente operou no estratégico (E1).

O discurso da entrevistada revela a preocupação em não fazer contribuições sociais e ambientais que apenas reflitam crenças e valores pessoais dos gestores, mas que estejam vinculadas a objetivos bem formulados e alinhados à estratégia organizacional, conforme defendido por Porter e Kramer (2006). A empresa mostra reconhecer que a simples inclusão de programas de responsabilidade social não é suficiente para gerar valor à empresa, mas quando a relação entre RSC e ganho econômico é cuidadosamente desenhada. A seguir, as três práticas consideradas estratégicas para empresa são descritas:

\section{Educação Financeira}

O banco declara que considera a educação financeira um assunto estratégico, pois acredita 
que o desequilíbrio nas finanças pessoais poderia comprometer a capacidade de pagamento, prejudicando os cidadãos, as empresas e o país. Com o endividamento as pessoas deixarão de consumir, as indústrias desacelerarão suas atividades, deixarão de gerar empregos e pagar impostos. O mesmo vale para os empreendimentos de pequeno porte, que sem uma boa gestão orçamentária, não terão recursos para investir em novas frentes de mercado, limitando seu crescimento (RELATÓRIO DE SUSTENTABILIDADE, 2012). Essa lógica, apresentada pela empresa, refere-se à preocupação com seu contexto competitivo, pois as condições externas exercem influência sobre os resultados da empresa (PORTER; KRAMER 2006).

\section{Riscos e Oportunidades Socioambientais}

A empresa possui uma política que formaliza os critérios socioambientais adotados no processo de crédito para empresas. Para analisar e liberar recursos, além dos aspectos convencionais, como finanças, gestão e governança, são observadas as práticas das companhias com relação aos direitos humanos, meio ambiente e biodiversidade, entre outros. Alguns exemplos de quesitos avaliados são: utilização de mão de obra infantil de forma prejudicial e/ou exploratória; empresas incluídas no cadastro de empregadores que tenham mantido trabalhadores em condições análogas à de escravo e questionário de autodeclaração socioambiental, respondido pelas empresas. Além disso, nas operações de project finance acima de US\$ 10 milhões, a empresa segue os Princípios do Equador (RELATÓRIO DE SUSTENTABILIDADE, 2008, 2012).

A entrevistada E1 aborda essa lógica:

É visível que não existe um jeito de não olhar essas questões (socioambientais), porque se você está financiando um posto de gasolina, por exemplo, e ele tiver uma multa porque contaminou o solo [...] ele vai parar de pagar o empréstimo, não por uma questão ambiental, mas porque ele foi multado. A atividade foi interrompida e, portanto, ele não gera mais caixa. É um problema financeiro que começou por um problema ambiental.

É importante destacar que a prática de inclusão de riscos e critérios socioambientais no processo de crédito para empresas realizada pelo banco está de acordo com o movimento de crescente regulamentação de mecanismos para incluir a responsabilidade socioambiental no SFN (BANKTRACK; AMIGOS DA TERRA, 2012).

Além da diminuição de riscos, a empresa identifica oportunidades para o desenvolvimento de 
novos produtos e serviços, em áreas relacionadas com a responsabilidade socioambiental. Por exemplo, existem financiamentos ligados a projetos de redução de resíduos sólidos e emissão de efluentes, troca de combustível líquido por gás natural, financiamentos para aquisição de veículos para pessoas com deficiência e financiamentos para o plantio de eucalipto para pequenos produtores rurais. Esses financiamentos mostram que, conforme relatório do IFC (2007), os bancos estão, cada vez mais, se deslocando da postura de evitar riscos para a postura de criar oportunidades, ou seja, saindo da postura defensiva para uma postura em que a responsabilidade social e ambiental é vista como uma vantagem e uma oportunidade de crescimento.

\section{Diálogo e Transparência}

Transparência nos mercados financeiros é uma das maiores demandas representadas pelas organizações que monitoram a ação dos bancos, como Banktrack, Bank Information Center e Bretton Woods Projects, que manifestaram fortes críticas à falta de transparência dos bancos, logo após os primeiros impactos da crise financeira de 2008. A transparência nos mercados financeiros refere-se à possibilidade de mapear os fluxos dos recursos financeiros e é uma condição para o seu próprio funcionamento, uma vez que permite aos agentes financeiros avaliar e gerenciar os potenciais riscos e retornos de suas operações. Além disso, reflete uma preocupação com as consequências econômicas das atividades financeiras, como a produção de emprego e, mais recentemente, a qualidade de vida das pessoas e o meio ambiente (MAGALHÃES, 2010).

Seguindo essa lógica, a empresa estudada declara que a perenidade dos seus negócios depende da maneira como é feita a interação com seus colaboradores, clientes, acionistas e a sociedade. Para construir relações de longo prazo entre a empresa e esses públicos e trazer resultados para todos os envolvidos, afirma ser necessário ouvir e entender suas demandas e comunicar os compromissos e resultados dos negócios, com clareza e transparência (RELATÓRIO DE SUSTENTABILIDADE, 2012). Essa declaração está de acordo com a afirmação de Clarkson (1995) que defende que a continuidade da empresa depende de sua habilidade em equilibrar as relações com os diversos stakeholders.

\subsubsection{Diálogo com stakeholders}


Outra condição proposta no modelo do presente estudo para o alinhamento estratégico da RSC é o diálogo com os stakeholders. Entre 2010 e 2011, período em que os processos e práticas de RSC foram redesenhados, a empresa realizou entrevistas com o presidente do conselho de administração, o presidente executivo, todos os vice-presidentes e diversos diretores. Em 2011, foi feita a análise da visão e da cultura, do propósito da marca, das políticas e regulamentos do banco, além dos compromissos e pactos voluntários dos quais a empresa é signatária. Depois disso, foram feitos cinco workshops com representantes de áreas do banco; apresentação do resultado do trabalho aos diretores para garantir a integração à agenda estratégica dos negócios; validação da proposta em um novo painel de diálogo com especialistas externos; validação pelo comitê de sustentabilidade; validação final pelo comitê executivo (RELATÓRIO DE SUSTENTABILIDADE, 2011). A Figura 3 resume esse processo de escolha das novas iniciativas de RSC.

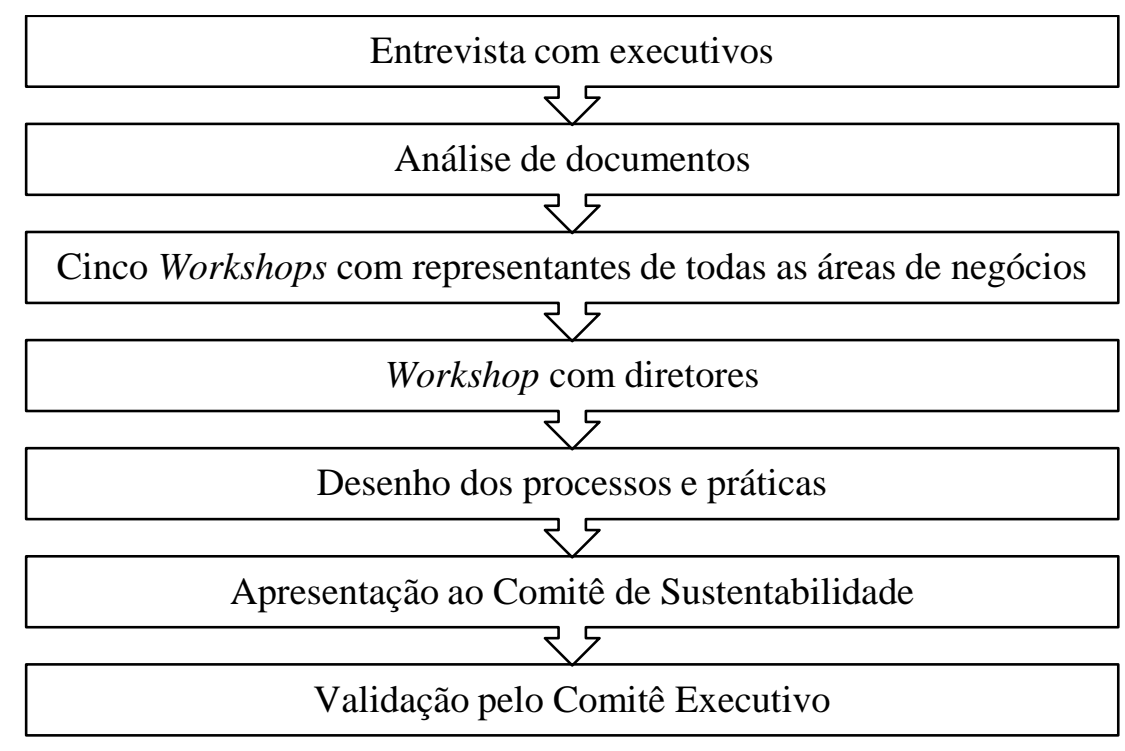

Figura 3 - Processo de escolha dos processos e práticas de RSC.

FONTE: Adaptado de Relatório de Sustentabilidade, 2011.

A entrevistada E2 explica que, até 2009, havia oito temas considerados prioritários (conforme Figura 2). Com o tempo, surgiu a necessidade de discutir como a empresa deveria atuar com relação a eles. Para isso, iniciou-se esse processo de consulta de seus stakeholders. O feedback recebido foi de que os oito temas abrangiam coisas muito distintas e que o banco deveria priorizar e focar em questões mais ligadas ao seu negócio (E2). Os stakeholders consultados afirmaram que alguns temas eram bastante transversais - como engajamento dos 
stakeholders - enquanto outros eram muito específicos - como diversidade, por exemplo (E2). Pode-se observar, portanto, que a empresa criou um diálogo preliminar com seus stakeholders, conforme defendido por Molteni (2006). Isso foi feito por meio de painéis e workshops realizados durante o processo de escolha dos novos processos e práticas de RSC.

\subsubsection{Envolvimento das áreas funcionais}

Foi possível observar também, na empresa, a preocupação com o envolvimento das áreas funcionais nas práticas e processos de RSC. Para a integração e o aprofundamento dos três temas estratégicos nas diversas áreas, foram estabelecidas quatro frentes viabilizadoras estruturas internas da organização, transversais a todas as áreas que funcionam como engrenagens. As frentes viabilizadoras são alavancas internas que dão sustentação às diversas iniciativas desenvolvidas em todas as áreas do banco para que os focos estratégicos estejam sempre conectados às estratégias de negócio. As quatro frentes viabilizadoras são: governança e gestão; incentivos; eficiência; cultura (RELATÓRIO DE SUSTENTABILIDADE, 2012), conforme mostrado na Figura 2.

Segundo o Relatório Anual de Sustentabilidade (2010), em 2009, a empresa intensificou um processo de incorporação da RSC em todas as suas áreas, com o objetivo de alcançar um alinhamento transversal das práticas em suas operações e processos. Para atingir esse objetivo, o banco afirmou que:

[...] se apoia em estratégias claras e bem definidas de atuação, em políticas que norteiam o relacionamento com os stakeholders, na transparência e ética nos negócios e na prestação de contas, além de uma sólida governança corporativa que insere progressivamente os conceitos da sustentabilidade em suas decisões (RELATÓRIO DE SUSTENTABILIDADE, 2010, p. 30).

A entrevistada E3, ao falar da integração da RSC nas diversas práticas e processos, afirma que:

A gente tem se detido mais em mexer nas engrenagens de fazer banco, olhando como essas questões conversam com cada uma dessas engrenagens [...] Gerir essas questões [...] dentro do banco como um todo. Isso não é uma responsabilidade da área de sustentabilidade, mas tem que ser uma responsabilidade das áreas do banco, patrocinada por uma área (de sustentabilidade). A mudança é executada dentro das próprias áreas, porque são elas que têm que se adaptar. Se não eu crio o banco sustentável na área de sustentabilidade e o banco continua [...] 
3.3.4 Envolvimento da alta gestão e Monitoramento e Gerenciamento dos resultados

Com relação ao envolvimento da alta gestão e monitoramento e gerenciamento dos resultados, destaca-se que, em 2012, a empresa construiu o painel de gestão de sustentabilidade, que permite o acompanhamento detalhado pelo comitê executivo das diversas iniciativas ligadas à RSC desenvolvidas pelo banco. O painel foi formado com base nos três temas considerados estratégicos e incorpora os indicadores utilizados pelos índices DJSI e pelo ISE como mecanismo para avaliar as áreas em que há avanços e as que ainda exigem ações de melhoria, contribuindo com a evolução em cada área (E2).

A partir dessa análise, surge o painel de projetos, com indicadores de desempenho vinculados a indicadores internos e externos, como do Global Reporting Initiative (GRI), que são utilizados para monitorar o andamento dos projetos. Esse processo se iniciou quando a área de sustentabilidade apresentou para o comitê executivo a situação da empresa com relação ao DJSI, ao ISE e às escolhas dos processos e práticas feitas pela empresa. A partir disso, o comitê executivo instruiu que os projetos fossem alinhados com cada área de negócio. Então, os gestores de sustentabilidade, em conjunto com os executivos das áreas, determinam o que deve ser feito, o que precisa ser melhorado e o que já está adequado, ou seja, o conjunto de projetos e iniciativas dentro das áreas que geram o painel, que funciona como um grande sinaleiro e é reportado ao comitê executivo duas vezes por ano (E2).

A recente criação do painel de gestão mostra a preocupação em monitorar a RSC nas diferentes áreas de negócio, o que, de acordo com Clarkson (1995), indica que a empresa atribui importância e relevância ao tema para justificar o acompanhamento.

\subsubsection{Síntese dos Principais Resultados}

A síntese dos resultados encontrados, organizados por: 1) condições para o alinhamento propostas no framework (Figura 1), 2) evidências observadas em campo e 3) Processos e práticas adotados para o alinhamento, é mostrada no Quadro 3.

Quadro 3 - Síntese dos Resultados. 


\begin{tabular}{|c|c|c|}
\hline $\begin{array}{c}\text { Condições para o } \\
\text { Alinhamento }\end{array}$ & Evidências das Condições & Processos e Práticas adotados \\
\hline $\begin{array}{c}\text { Práticas que } \\
\text { melhoraram o } \\
\text { contexto competitivo }\end{array}$ & $\begin{array}{l}\text { Incentivo e priorização de } \\
\text { projetos com atributos de RSC; } \\
\text { preservação do meio ambiente; } \\
\text { melhoria na educação } \\
\text { financeira. }\end{array}$ & $\begin{array}{l}\text { Inclusão da variável socioambiental na } \\
\text { avaliação de crédito, investimentos e } \\
\text { novos produtos e serviços; Avaliação } \\
\text { sistemática de despesas; Redução de } \\
\text { desperdícios, otimização de recursos e } \\
\text { sinergia entre as atividades operacionais; } \\
\text { Educação Financeira para funcionários, } \\
\text { clientes e não clientes }\end{array}$ \\
\hline $\begin{array}{l}\text { Vínculo das práticas } \\
\text { sociais com a } \\
\text { atividade principal da } \\
\text { empresa. }\end{array}$ & $\begin{array}{c}\text { Criação de três focos } \\
\text { estratégicos (Educação } \\
\text { Financeira; Riscos e } \\
\text { Oportunidades Socioambientais; } \\
\text { Diálogo e Transparência) } \\
\end{array}$ & $\begin{array}{l}\text { Redesenho dos processos e práticas de } \\
\text { RSC (Figura 4). }\end{array}$ \\
\hline $\begin{array}{l}\text { Diálogo com } \\
\text { stakeholders }\end{array}$ & $\begin{array}{l}\text { Diálogo com todos os } \\
\text { stakeholders da empresa: } \\
\text { acionistas, funcionários, } \\
\text { clientes, sociedade e governo. }\end{array}$ & $\begin{array}{l}\text { Painéis, workshops e pesquisas com } \\
\text { stakeholders internos e externos; } \\
\text { Criação de canais de comunicação com } \\
\text { funcionários, clientes e sociedade; } \\
\text { Consulta a especialistas }\end{array}$ \\
\hline $\begin{array}{l}\text { Envolvimento das } \\
\text { áreas funcionais. }\end{array}$ & $\begin{array}{c}\text { Criação das quatro frentes } \\
\text { viabilizadoras: Governança e } \\
\text { Gestão; Incentivos; Eficiência; } \\
\text { Cultura. }\end{array}$ & $\begin{array}{c}\text { Criação da Política de Sustentabilidade; } \\
\text { Participação da área de Sustentabilidade } \\
\text { em outras instâncias do banco, como o } \\
\text { Comitê de Excelência do Atendimento, } \\
\text { o Comitê de Risco Socioambiental e o } \\
\text { Comitê de Avaliação de Processos e } \\
\text { Produtos. }\end{array}$ \\
\hline $\begin{array}{l}\text { Envolvimento da Alta } \\
\text { Gestão }\end{array}$ & $\begin{array}{c}\text { Criação da Governança de } \\
\text { Sustentabilidade; Participação } \\
\text { do Comitê Executivo }\end{array}$ & $\begin{array}{l}\text { A governança de Sustentabilidade insere } \\
\text { progressivamente os conceitos de RSC } \\
\text { em suas decisões; Comitê Executivo } \\
\text { avalia os resultados duas vezes por ano. }\end{array}$ \\
\hline $\begin{array}{l}\text { Monitoramento e } \\
\text { gerenciamento dos } \\
\text { resultados }\end{array}$ & Criação do Painel de Gestão & $\begin{array}{c}\text { Observação de práticas utilizadas no } \\
\text { mercado; Comparação com indicadores } \\
\text { dos índices de sustentabilidade ISE e } \\
\text { DJSI e do GRI. }\end{array}$ \\
\hline
\end{tabular}

É importante destacar que as entrevistadas levantaram algumas barreiras para a integração da RSC nas atividades da empresa. As entrevistadas acreditam que a falta de ferramentas e de conhecimento são as principais dificuldades. Outra barreira levantada é o problema de escala. Não há um sistema com informações padronizadas e legalmente reconhecidas de possíveis irregularidades socioambientais dos clientes que possa ser consultado em grande escala. Essa falta de informações acaba exigindo um grande volume de verificações, o que não é viável para empresas com milhões de clientes.

Não tem ferramenta pronta no mercado, por exemplo, com relação à avaliação do risco socioambiental [...] E a métrica por trás disso? Como é que eu avalio isso? Não tem no mercado. Você não compra em uma consultoria. Não existe consultoria que tenha isso. Não existe um sistema que você compra, acopla e sai fazendo. Você tem que treinar as pessoas, porque ninguém foi treinado para isso. Você tem que desenvolver a métrica, 
testar a métrica para ver se funciona. Tudo tem que ser feito. Então o "como" é um aprendizado (E2).

\section{CONSIDERAÇÕES FINAIS}

Apesar da falta de consenso sobre os impactos financeiros da RSC, a ideia de que a organização deve se preocupar com seu desempenho social e não somente com seu desempenho financeiro parece estar fortalecida no meio acadêmico e empresarial. A principal questão levantada agora é "como" e "quando" incorporar as questões sociais nos negócios.

O presente estudo buscou analisar quais processos e práticas favorecem o alinhamento estratégico da RSC. Para atingir esse objetivo, foi proposto um modelo de alinhamento, e em seguida, esse modelo foi aplicado empiricamente em um estudo de caso de uma empresa com alta CSP e alta CFP. Foi encontrado, tanto na literatura quanto no estudo de campo, que algumas condições são necessárias para esse alinhamento. As condições são: melhora do contexto competitivo; vínculo das práticas de RSC com a atividade principal da empresa; diálogo com stakeholders; envolvimento da alta gestão; envolvimento das áreas funcionais; monitoramento e gerenciamento de resultados.

O estudo contribui com o aprofundamento da discussão sobre a complexa relação entre CSP e CFP e sobre as implicações estratégicas da RSC. A segunda contribuição do estudo refere-se à criação de um modelo para análise de alinhamento estratégico de RSC. Além disso, o estudo pode auxiliar empresas que desejam promover o alinhamento entre RSC e estratégia e buscar ganhos financeiros associados a ganhos sociais. $\mathrm{O}$ estudo também levanta algumas barreiras para a gestão de assuntos de RSC. A principal delas é a falta de conhecimento e ferramentas práticas disponíveis para lidar com o assunto.

\section{REFERÊNCIAS}

AMIGOS DA TERRA. Introdução ao setor bancário e suas práticas de sustentabilidade. São Paulo: Amigos da Terra, dez. 2012. Disponível em:

<http://www.banktrack.org/manage/ems_files/download/introducao_ao_setor_bancario_brasil eiro_e_suas_praticas_de_sustentabilidade/121219_brasil_setor_bancario_e_sustentabilidade_ 2012_12_0.pdf>. Acesso em: 05 mar. 2013.

BACKHAUS, K. B.; STONE, B. A.; HEINER, K. Exploring the relationship between corporate social performance and employer attractiveness. Business \& Society, Thousand Oaks, v. 41, n. 3, p. 292-318, 2002. 
BLACK, T. Doing quantitative research in the social sciences: an integrated approach to research design, measurement and statistics. Thousand Oaks: Sage, 1999.

BRANCO, M. C.; RODRIGUES, L. L. Corporate Social Responsibility and Resource-Based Perspectives. Journal of Business Ethics, New York, v. 69, n. 2, p. 111-132, 2006.

BURKE, L.; LOGSDON, J. M. How corporate social responsibility pays off. Long Range Planning, Amsterdam, v. 29, p. 495-502, 1996.

CARROLL, A. B. A Three-Dimensional Conceptual Model of Corporate Social Performance. Academy of Management Review, Briarcliff Manor, v. 4, n. 4, p. 497-505, 1979.

CARROLL, A. B. The pyramid of corporate social responsibility: Toward the moral management of organizational stakeholders. Business Horizons, v. 34, n. 4, p. 39-48, 1991.

CARROLL, A. B. A history of corporate social responsibility: concepts and practices. In: CRANE, A. et al. The Oxford Handbook of Corporate Social Responsibility. New York: Oxford University Press, 2008.

CHANDLER, A. Strategy and structure. Massachusetts: MIT Press, 1962.

CLARKSON, M. B. A Stakeholder Framework for Analyzing and Evaluating Corporate Social Performance. Academy of Management Review, Briarcliff Manor, v. 92, p. 105-108, 1995.

CRANE, A. et al. The Corporate Social Responsibility Agenda. In: The Oxford Handbook of Corporate Social Responsibiliy. New York: Oxford University Press, 2008.

DAVIS, K. Five Propositions for Social Responsibility. Business Horizons, Amsterdam, n. 18, n. 3, p. 19-24, 1975.

DeBAKKER, G. A. GROENWEGEN, P. DEN HOND, F. A Bibliometric analysis of 30 years of research and theory on Corporate Social Responsibility and Corporate Social Performance. Business and Society, Thousand Oaks, v. 44, n. 3, p. 283-317, 2005.

ECONOMATICA. Disponível em: <http://www.economatica.com>. Acesso em: 20 abr. 2013

EISENHARDT, K. M.; GRAEBNER, M. E. Theory building from cases: opportunities and challenges. Academy of management journal, Briarcliff Manor, v.50, n. 1, p. 25-32, 2007.

FOMBRUN, C; GARDBERG, N. A.; BARNETT, M. L. Opportunity Platforms and Safety Nets: Corporate Citizenship and Reputational Risk. Business and Society Review, New York, v. 105, n. 1, p. 85-106, 2000.

FREDERICK, W. C. The growing concern over Business Responsibility. California Management Review, Berkeley, v. 29, p. 99-114, 1960. 
FRIEDMAN, M. The social responsibility of business is to increase its profits. New York Times Magazine, New York, v. 13, 1970.

GVCES - CENTRO DE ESTUDOS EM SUSTENTABILIDADE. Índice de Sustentabilidade Empresarial. Disponível em: <https://www.isebvmf.com.br>. Acesso em: 20 abr. 2013.

HENDERSON, J. C., VENKATRAMAN, N. Strategic Alignment: A framework for strategic information technology management. Cambridge, MA: MIT Press, 1989.

HUSTED, B. W. A contingency theory of corporate social performance. Business and Society, Thousand Oaks, v. 39, n. 1, p. 24-48, 2000.

HUSTED, B. W; SALAZAR, J. D. J. Taking Friedman seriously: maximizing profits and social performance. Journal of Management Studies, New York, v. 43, n. 1, p. 75-91, 2006.

IFC - Banking on Sustainability: Financing Environmental and Social Opportunities in Emerging Markets. Washington: International Finance Corporation, 2007.

JONES, M. T. Corporate Social Responsibility Revisited, Redefined. California Management Review, Berkeley, v. 22, n. 2, p. 59-67, 1980.

KAPLAN, R. S.; NORTON, D. P. Strategy Maps: Converting Intangible Assets into Tangible Outcomes. Boston, MA: Harvard Business Press Books, 2004.

KARNANI, A. Doing Well by Doing Good: The Grand Illusion. California Management Review, Berkeley, v. 53, n. 2, p. 69-86, 2011.

KURUCZ, E. C. The business case for corporate social responsibility. In: CRANE, A. et al. The Oxford Handbook of Corporate Social Responsibility. New York: Oxford University Press, 2008.

LOCKETT, A.; MOON, J.; VISSER, W. Corporate Social Responsibility in Management Research: Focus, Nature, Salience and Sources of Influence. Journal of Management Studies, New York, v. 43, n. 1, p. 115-36, 2006.

MARCONDES, A. W.; BACARJI, C. D. ISE - Sustentabilidade no Mercado de Capitais. São Paulo: Report Editora, 2010.

MARGOLIS, J.; WALSH, J. Misery loves company: rethinking social initiatives by business. Administrative Science Quarterly, Thousand Oaks, v. 48, n. 2, p. 268-305, june 2003.

McWILLIAMS, A.; SIEGEL, D. S. Corporate social responsibility: a theory of the firm perspective. Academy of Management Review, Briarcliff Manor, v. 26, n. 1, p. 117-127, 2001.

McWILLIAMS, A.; SIEGEL, D. S; WRIGHT, P. M. Corporate Social Responsibility: Strategic Implications. Journal of Management Studies, New York, v. 43, n. 1, p. 1-18, 2006. 
McWILLIAMS, A.; SIEGEL, D. S. Creating and Capturing Value: Strategic Corporate Social Responsibility, Resource-Based Theory, and Sustainable Competitive Advantage. Journal of Management, Thousand Oaks, v. 37, n. 5, p. 1480-1495, 2011.

MAGALHÃES, R. S. Lucro e reputação: interações entre bancos e organizações sociais na construção das políticas socioambientais. 2010. Tese (Doutorado em Ciência Ambiental) - Programa de Pós-Graduação em Ciência Ambiental da Universidade de São Paulo, São Paulo, 2010.

MICHALOS, A. Issues for business ethics in the nineties and beyond. Journal of Business Ethics, New York, v. 16, n. 3, p. 219-231, 1997.

MOLTENI, M. The social-competitive innovation pyramid. Corporate Governance, New York, v. 6, n. 4, 2006.

NASCIMENTO, L. F.; LEMOS, A. D. C.; MELLO, M. C. A. Gestão Socioambinetal Estratégica. Porto Alegre: Bookman, 2008.

ORLITZKY, M.; SCHMIDT, F. L.; RYNES, S. L. Corporate Social and financial performance: a meta-analysis. Organization Studies, Thousand Oaks, v. 24, n. 3, p. 403-441, 2003.

PORTER, M. E.; KRAMER, M. R. The competitive advantage of corporate philanthropy. Harvard Business Review, Cambridge, v. 80, n. 12, p. 57-68, 2002.

PORTER, M. E.; KRAMER, M. R. Strategy and society: the link between competitive advantage and corporate social responsibility. Harvard Business Review, Cambridge, v. 84, n. 12, p. 78-92, 2006.

RELATÓRIO ANUAL DE SUSTENTABILIDADE (2008, 2009, 2010, 2011, 2012).

Disponível em:

<http://www.itauunibanco.com.br/relatoriodesustentabilidade/2012/pt/ra/index.html $>$. Acesso em: 15 mar. 2013.

RUSSO, M. V.; FOUTS, P. A. A Resource-Based Perspective on Corporate Environmental Performance and Profitability. Academy of Management Journal, Briarcliff Manor, v. 40, n. 3, p. 534-559, 1997.

STAKE, R. E. Case Studies. In: DENZIN, N. K.; LINCOLN, Y. S. (Org.). Handbook of qualitative research, Thousand Oaks, p. 435-454, 1994.

SURROCA, J.; TRIBÓ, J. A.; WADDOCK, S. Corporate responsibility and financial performance: the role of intangible resources. Strategic Management Journal, Hoboken, v. 31, n. 5, p. 463-490, 2010.

ULLMANN, A. Data in search of a theory: A critical examination of the relationship among social performance, social disclosure, and economic performance. Academy of Management Review, Hoboken, 10: 540-577, 1985.

WADDOCK, S.A. Parallel universes: companies, academics, and the progress of corporate 
citizenship. Business and Society Review, Hoboken, v. 109, n.1, p. 5-42, 2004.

WOOD, D. J. Corporate Social Performance Revisited. Academy of Management Review, Briarcliff Manor, n. 16, p. 66-73, 1991.

YIN, R. K. Estudo de Caso: planejamento e métodos. 4 ed. Porto Alegre: Bookman, 2010. Tradução: Ana Thorell. 